Goudet, Anna et Catherine Paquette. « Quand la « voix » des nouveaux·elles arrivant·e·s est exprimée et entendue grâce à la photographie : réflexions sur la méthode de recherche Photovoice ». Nouvelle Revue

\title{
Quand la « voix » des nouveaux·elles arrivant·e·s est exprimée et entendue grâce à la photographie : réflexions sur la méthode de recherche Photovoice
}

\author{
Anna Goudet \\ Catherine Paquette \\ Institut National de la recherche scientifique \\ Centre Urbanisation Culture Société \\ Canada
}

\section{Introduction}

Censés représenter le monde « tel qu'il est », les clichés photographiques ont accompagné le travail de plusieurs générations d'anthropologues et permis de poser un regard différent et détaillé sur leurs thèmes d'études en terrain inconnu. Aujourd'hui, la photographie et les méthodes visuelles se taillent tranquillement une place légitime dans les méthodologies de recherche en sciences sociales. L'une de ces méthodes consiste à transférer l'appareil photo des mains des chercheur·e·s à celui des participant·e·s, afin de les inviter à élaborer leurs propres représentations visuelles : le Photovoice. Cette méthode - qui repose sur la prise de photographies par les participant·e.s et sur un retour sur celles-ci en entrevue individuelle ou de groupe - est en effet de plus en plus utilisée en recherche-action auprès de communautés généralement exclues des processus décisionnels ou systémiquement sous-représentées afin de co-construire des connaissances fines et localisées, à partir de leur point de vue. La méthode Photovoice est notamment appliquée dans le champ de la santé, du travail social et des études urbaines, pour nourrir des projets de développement prenant appui sur la consultation des populations locales (Houle et al., 2016 ; Lofton et Grant, 2020).

Cet article propose de réfléchir à la notion de "voix », à partir d'un projet de recherche qui a reposé sur une démarche Photovoice pour saisir les perceptions et expériences de l'accueil de nouveaux et nouvelles arrivant·ess dans deux arrondissements de Montréal. Cette étude, réalisée en collaboration avec des organismes communautaires de ces arrondissements, nous a permis de saisir six dimensions qui contribuent au caractère accueillant de leur quartier selon les personnes immigrantes qui y ont participé : la fonctionnalité, l'esthétisme, le soutien, la sociabilité, la participation sociale et la présence de repères symboliques (Goudet et al., 2021). L'expérience et l'importance accordée à ces dimensions varient selon les caractéristiques sociales des répondant·ess (notamment leur genre, leur parcours migratoire, leurs situations socioéconomique, professionnelle et familiale, leur maîtrise de la langue, etc.). Ce projet avait entre autres pour objectif de nourrir la réflexion des organismes locaux dans le cadre de leur démarche de mise en place de collectivités accueillantes, et pour se faire de se porter à l'écoute de la « voix » de personnes immigrantes de ces territoires.

Dans le présent article, nous souhaitons effectuer un retour réflexif sur notre approche méthodologique, en revenant sur certains résultats et propos tenus par les participant·e·s en entrevue. Nous mettons de côté une importante partie de notre première analyse pour nous concentrer sur deux éléments en particulier relatifs à notre démarche Photovoice. D'une part, un résultat d'analyse : l'importance donnée par les participant·e·s à la valeur esthétique et aux émotions ressenties dans les espaces jugés accueillants et exprimées par les photographies. Ce résultat n'était pas attendu, puisque cette dimension n'a pas été abordée par la littérature sur les collectivités accueillantes (Esses et al., 2010) qui avait guidé certaines questions de départ. D'autre part, les modes de participation des personnes rencontrées : ils et elles se sont adapté $\cdot e \cdot s$ et approprié la démarche à leur manière, ce qui nous amènera à discuter de la démarche Photovoice comme espace de co-construction des données et de la réalité urbaine. À la lumière de ces éléments, nous apporterons quelques pistes de réponses aux questions suivantes : quels avantages et limites a posé la méthode Photovoice pour l'expression de la parole de personnes immigrantes dans le cadre de notre recherche exploratoire? De quelle façon cette démarche a-t-elle permis de faire entendre leur voix au sujet de certaines de leurs préoccupations à l'échelle de la communauté locale de leur quartier? Ces questionnements nous amèneront à soulever certains enjeux épistémologiques relatifs à l'utilisation de la méthode Photovoice et à l'espace de co-création des données qui peut en résulter.

Nous commencerons par présenter la démarche Photovoice, puis, brièvement, le projet de recherche au cours duquel nous y avons eu recours en 2019 et 2020. Nous discuterons ensuite, en trois parties, du potentiel et des défis de cette méthode pour faire émerger la « voix » de personnes immigrantes ${ }^{1}$ dans une recherche sociale en milieu urbain. 
Goudet, Anna et Catherine Paquette. « Quand la « voix » des nouveaux·elles arrivant·e·s est exprimée et entendue grâce à la photographie : réflexions sur la méthode de recherche Photovoice ». Nouvelle Revue

Synergies Canada, № 15 (2022)

\section{La méthode Photovoice et la recherche-action}

À l'origine, la méthode Photovoice repose sur trois courants théoriques : les théories du développement de la réflexion critique, les théories féministes et la photographie documentaire (Wang et Burris, 1997). Si cette approche a émergé dans les domaines de la santé, elle s'est depuis étendue à de multiples champs d'études, sur de nombreux sujets et auprès d'une variété de population. Son usage trouve toutefois un écho plus particulier auprès des populations plus défavorisées, comme des Inuits (Budach et Patrick, 2011), des femmes monoparentales (Valiquette-Tessier et al., 2015), des personnes âgées (Ronzi et al.,2016) et des résident·e·s d'habitations à loyer modique (HLM) (Houle et al., 2016). La méthode Photovoice est également de plus en plus utilisée pour identifier des enjeux et perceptions liés aux aspects sociaux et au cadre bâti de certains environnements, en particulier les quartiers et environnements résidentiels (Houle et al., 2016), ce qui fait écho à notre démarche sur le territoire montréalais. Lorsqu'elle est employée dans le cadre de questionnements au sujet de l'environnement, la photographie permet aussi de faire émerger des perspectives sociales sur les espaces physiques (Nykiforuk et al., 2011).

Concrètement, elle consiste à combiner la prise de photographies par les participant $\cdot e \cdot s$ et des entretiens avec les participant $\cdot \mathrm{e} \cdot \mathrm{s}$. Les participant $\cdot \mathrm{e} \cdot \mathrm{s}$ documentent eux.elles-mêmes leurs perceptions et expériences à travers la représentation visuelle. Contrairement à d'autres méthodes visuelles, il ne s'agit toutefois pas de considérer que les photographies contiennent les données. Dans la démarche Photovoice, la photographie est le point de départ pour générer une conversation, menant à un processus réflexif et approfondi au sujet de l'expérience en question lors de l'entretien: « the conversations that centre on the image generate the data, encouraging both research participant and researcher to be more reflexive in their thoughts and feelings about the research questions and indeed tangential issues too »(Warren, 2005, p. 867). Les recherches Photovoice reposent généralement sur la participation de petits groupes de personnes en entrevue collective, où les séries d'images des participant·e·s servent à guider la discussion (Wang et Burris, 1997).

La méthode amène ainsi les participant·e·s à occuper un rôle actif dans la recherche (ils et elles produisent les images qui deviendront centrales à la recherche), plutôt que de les limiter à répondre à des questions, comme lors d'entretiens semi-dirigés. Les résultats issus à la fois de ces conversations et des ensembles d'images font ensuite l'objet d'expositions ou de communications publiques qui sont à leur tour le point de départ de discussions et de changements dans la communauté (Ronzi et al., 2016, p. 733 ; Lofton et Grant, 2020), d'où l'emploi de plus en plus fréquent de la démarche en recherche-action.

En décrivant les enjeux, les ressources, les épreuves et les parcours qui ponctuent leur expérience quotidienne au moyen de la photographie, il est attendu que les participant.e.s procèdent à une « re-qualification " (Payet et al., 2008) de leur voix (Evans-Agnew et Rosemberg, 2016). Par la suite, la diffusion conjointe de ces photographies et des résultats de recherche contribue à promouvoir le dialogue critique et la création de connaissances en lien avec les préoccupations de la communauté, en plus de rejoindre les décideur·euse-s de la communauté locale (Wang et Burris, 1997, p. 37). Pour Nykiforuk et ses collègues, le rôle principal occupé par les participant $\cdot$ - $s$ dans le processus du Photovoice, où il leur est demandé de documenter visuellement leur perception, a également l'avantage de leur prouver qu'ils elles sont des membres importants de l'équipe de recherche (Nykiforuk et al., 2011, p. 105), ce qui accentue la portée de leur voix auprès de la communauté locale qui sera témoin des résultats.

Dans le cadre de notre projet, les photographies et les discussions qui en ont résulté lors des entretiens ont servi à identifier des éléments appréciés ou dépréciés par les participants dans leur quartier. Mais plus encore, nous avons remarqué que la démarche Photovoice a aussi donné lieu à différentes formes de participation, et même d'appropriation de cet outil qu'est la photo, par les personnes rencontrées, durant la démarche de recherche. Les corpus de photographies variés, ainsi que les remarques ou attitudes tout à fait contrastées à propos de la prise de photographie, donnent des indices quant à la liberté prise par les participants dans leur façon de répondre à nos questionnements sur leur milieu de vie résidentiel, et ce, indépendamment de leurs préoccupations vis-à-vis du quartier et la communauté locale.

\section{Le projet « Perception et accueil des personnes immigrantes dans les quartiers montréalais : un projet Photovoice »}

L'étude que nous avons réalisée en 2019 et 2020 s'inscrit dans le cadre plus large d'une recherche-action de longue haleine qui documente l'initiative «Vivons nos quartiers » (TCRI et Centraide). Ce projet qui rassemble 
Goudet, Anna et Catherine Paquette. « Quand la « voix » des nouveaux·elles arrivant·e·s est exprimée et entendue grâce à la photographie : réflexions sur la méthode de recherche Photovoice ». Nouvelle Revue

Synergies Canada, № 15 (2022)

des organismes communautaires et institutions locales est né dans le contexte des arrivées nombreuses de personnes réfugiées et revendiquant l'asile dans le Grand Montréal (Québec, Canada), qui ont bouleversé les structures d'accueil et la société civile depuis 2015-2016. "Vivons nos quartiers » a pour objectif de favoriser la mise en place et le partage de pratiques d'inclusion à l'échelle des quartiers de Montréal, afin d'en faire des collectivités accueillantes (Désilets et Goudet, 2019). Après avoir observé certains volets de l'initiative (Désilets et Goudet, 2019; Goudet et al., 2021), l'équipe de recherche multidisciplinaire (sociologie, anthropologie, sciences politiques, études urbaines), intégrée au Partenariat Immigration et résilience en milieu urbain (BMRCIRMU, CRSH 2016-2021), a entrepris de suivre étroitement le déploiement de deux réseaux locaux de concertation portant sur les enjeux liés à l'immigration et le rapprochement interculturel. Ces réseaux ont émergé dans deux territoires montréalais, Ville-Émard/Côte-Saint-Paul (VECSP) et Mercier-Hochelaga-Maisonneuve (MHM), qui ne constituent pas des espaces traditionnels d'accueil de nouveaux arrivants, mais qui voient de plus en plus de personnes immigrantes s'y installer.

C'est dans l'objectif d'informer et d'alimenter les réflexions et actions de ces réseaux locaux, devenus partenaires de recherche, qu'a émergé la volonté d'explorer les perceptions et expériences de personnes immigrantes dans ces quartiers. Nous avons adopté la méthode Photovoice pour sonder le point de vue de personnes immigrantes au sujet des espaces qu'elles considèrent comme accueillants, qu'elles fréquentent, qu'elles apprécient et qu'elles évitent dans leur quartier. Le cadre conceptuel de ce projet de recherche repose sur une approche de la diversité " on the ground » (Belkhodja, 2009), telle qu'elle est vécue dans la vie quotidienne, ainsi que sur la notion de « collectivité accueillante » (Esses et al., 2010). Cette dernière propose un ensemble de critères pour favoriser l'attraction, la rétention et l'installation de personnes immigrantes en milieu urbain. Notre démarche se veut toutefois davantage inductive qu'évaluative, plaçant au cœur de l'analyse la perception des participant·e·s.

La méthode Photovoice nous a semblé être l'approche tout indiquée pour saisir les perceptions des personnes immigrantes, mais aussi les partager avec les comités-partenaires. Dès la mise en place du projet, nous avons discuté des objectifs et de la portée de la recherche en lien avec les attentes des organismes des arrondissements. Nous avons également planifié un événement de rétroaction dans chaque quartier, dans un lieu souvent nommé par les participant·e·s, qui se voulait tant un moment de diffusion des résultats du projet de recherche que de rencontres entre des citoyens résidents et des organismes du territoire. Néanmoins, la pandémie de COVID-19 a freiné nos élans, et ces événements ont dû être remplacés par des activités virtuelles, prenant la forme d'un échange plus classique de diffusion des résultats, mais conservant toutefois au cœur les photographies des participant $\cdot \mathbf{e} \cdot \mathrm{s}$.

Dans le cadre de notre projet de recherche, nous avons réalisé des entrevues Photovoice individuelles avec 15 participant·e·s, recruté·e·s par le biais d'organismes communautaires, les réseaux sociaux et le bouche-àoreille. L'échantillon n'est pas représentatif de la population des quartiers concernés, mais répond à l'objectif convenu avec les partenaires communautaires d'offrir un éventail de profils très variés. Ils et elles (10 femmes, 4 hommes, 1 personne non-binaire) sont établis à Montréal depuis 1 à 10 ans (en majorité depuis moins de 5 ans), proviennent de pays divers (France, Chine, Venezuela, Corée du Sud, République Démocratique du Congo, Mexique, Colombie, Algérie) et sont arrivé·e·s avec différents statuts migratoires (demandeur·euse·s d'asile, touristes en attente de régularisation, étudiant·e $s$ internationaux, détenteur.trice $s$ de permis vacancestravail, résident·e.s permanent·e.s). Cet échantillon hétérogène l'est aussi du point de vue du degré de vulnérabilisation possible de ces personnes, au regard de facteurs tels que leur situation socio-économique, leur statut d'immigration, la maîtrise limitée d'une langue officielle, la stigmatisation et la marginalisation. Notons également le profil de l'équipe de recherche, formée de deux étudiantes entre 25 et 30 ans, blanches, d'origine canadienne et française, qui nous permettra d'aborder notre positionnement.

Les personnes rencontrées ont été invitées à prendre une dizaine de photos, avec leur propre téléphone, des espaces du quartier qu'elles jugeaient accueillants, moins accueillants, ou qui avaient été significatifs dans leur expérience d'établissement. Pour chaque participant $e$, deux rencontres ont été organisées : une première fois avant la prise de photographie pour établir le contact, faire connaissance et expliquer le projet et ses consignes. Une à deux semaines plus tard, un second entretien (avec la même chercheuse) avait pour objet les photographies prises par le.la participant $e$. Cet entretien comportait des questions sur le type d'équipement et de ressources utilisés, leurs réseaux de sociabilité et leur sentiment d'appartenance au quartier. Précisons que nous avions pensé la grille d'entretien en fonction de plusieurs aspects suggérés par la notion de collectivité accueillante (Esses et al., 2010), mais que nous avons pris soin de laisser la présentation de chaque photo par le.la participant·e devenir le fil de la discussion lors des entretiens. 
Goudet, Anna et Catherine Paquette. « Quand la « voix » des nouveaux·elles arrivant·e·s est exprimée et entendue grâce à la photographie : réflexions sur la méthode de recherche Photovoice ». Nouvelle Revue

Synergies Canada, № 15 (2022)

Nous avons débuté ce projet avec l'hypothèse selon laquelle la méthode Photovoice allait permettre de créer un ensemble de données fines et localisées sur l'expérience et la perception des espaces du quartier. Cette hypothèse s'est confirmée, et les résultats ont même dépassé nos attentes. Ils nous ont en effet emmenées dans des registres différents de ceux que nous avions présupposés lors de notre recension de la littérature sur les collectivités accueillantes (Esses et al., 2010).

Dans les sections qui suivent, nous aborderons deux effets particulièrement enrichissants de la méthode Photovoice pour le projet de recherche : le potentiel de l'expression artistique-émotionnelle et l'appropriation de la méthode par les participant·e·s. Nous reviendrons pour finir sur les défis que cette méthode soulève.

\section{Le choix de l'expression artistique-émotionnelle}

L'une des surprises apportées par cette méthode dans le cadre de ce projet de recherche réside dans la manière dont les participant·e·s se sont approprié la démarche d'un point de vue artistique et symbolique. L'importance donnée aux dimensions esthétique et symbolique par les participant·e.s a sans doute tout à voir avec l'utilisation de la photographie comme moyen d'expression. Nous supposons dans la section qui suit que les éléments du quartier choisis par les participant·e·s auraient été différents si la démarche n'avait compris que des entretiens semi-dirigés. En effet, certains lieux photographiés ont fait émerger des souvenirs, des états d'esprits que des questions alignées sur les critères des collectivités accueillantes n'auraient sans doute pas évoqués. Également, nous avons noté chez les participant·e·s que l'émotion véhiculée par l'image a pu leur servir à énoncer, ou à appuyer, d'autres remarques au sujet de leur quartier, ce qui laisse croire à une appropriation de la démarche par ces dernier.e.s. Pour résumer: d'une part leurs photographies ont mis de l'avant des aspects esthétiques, émotionnels ou symboliques de leur rapport au quartier, et d'autre part, leurs photographies ont permis de véhiculer des émotions liées à d'autres aspects de leur environnement résidentiel qu'ils apprécient ou qui les préoccupent.

\subsection{Une nouvelle dimension de l'accueil ?}

L'opportunité offerte par la photographie de s'exprimer autrement que par le langage, en représentant visuellement sa perception et ses expériences, s'est révélée particulièrement porteuse. L'image est bien connue pour sa capacité à exprimer « mille mots », alors que le langage présente souvent des limites à l'expression de sentiments plus profonds (Warren, 2005). Au-delà de l'avantage très pratique de faciliter le contournement des barrières de la langue, l'agencement de la parole et de l'image peut aussi jouer un rôle important dans la révélation d'éléments et concepts plus abstraits, en ce que la signification d'une image dépasse bien souvent le cadre matériel qu'elle représente :

The significance of the photograph goes beyond its literal signification by way of the routes of the primary processes ... [in] a succession of metonymies and metaphors which transpose the scene of the photograph to the spaces of the "other scene" of the unconscious, and also, most importantly, the scene of the popular preconscious : the scene of discourse, of language. (Warren, 2005, p. 875)

Ainsi, l'utilisation de la photographie, contrairement aux entretiens traditionnels où les propos sont plus souvent rapportés sous la forme de texte, permet la transmission directe/immédiate de l'opinion, de la subjectivité (Warren, 2005, p. 865) dans une façon que le texte ne permet pas, en plus de faire émerger différentes préoccupations lorsque les participant·e·s parlent d'une photographie qu'ils'elles ont prise et dont ils peuvent décoder les nuances. En effet, les images photographiques peuvent contribuer à communiquer le rapport au monde des participant $\cdot e \cdot s$ de façon plus précise, plus directe, ce qui amène à élever l'importance donnée à leur voix dans le processus de recherche (Warren, 2005). Le Photovoice « provides an opportunity to visually portray experiences and share personal knowledge about particular issues that may be difficult to express with words alone » (Nykiforuk et al. , 2011, p. 105).

Deux dimensions, en particulier, nous ont surpris dans la conception de nos participant $\cdot \mathbf{e} \cdot \mathbf{s}$ des espaces accueillants du quartier. D'une part, la dimension esthétique, et d'autre part, la dimension symbolique. Les participant·e·s ont ainsi exprimé leur perception d'un lieu accueillant comme pouvant être « joli », ou « vivant », ou faisant émerger des souvenirs. Ces dimensions ressortent à la fois par les photographies de certains lieux associés à ces émotions, et par les commentaires teintés de souvenirs et de sentiments qui accompagnent les photographies. Ces dimensions ne sont pas du tout abordées dans la liste des 17 caractéristiques proposées par Esses et al. (2010) pour mesurer le caractère accueillant des initiatives mises en place par les collectivités, 
Goudet, Anna et Catherine Paquette. « Quand la « voix » des nouveaux·elles arrivant·e·s est exprimée et entendue grâce à la photographie : réflexions sur la méthode de recherche Photovoice ». Nouvelle Revue

et qui font figure d'autorité dans les champs politique et académique canadiens sur le sujet. Néanmoins, elles sont également ressorties dans d'autres recherches qui explorent l'appropriation de la ville par la photographie (voir Alvir, 2013 notamment). Elle relève :

L'action de photographier a contribué à la définition des territoires et des frontières entre le visible et l'invisible, entre le cadre et le hors cadre. Elle nous a aidées à comprendre que l'espace investi n'est pas celui que l'on voit mais celui qui arrive à lever les frontières entre la vie d'avant et celle d'après, entre une présence ici et ailleurs. La photographie a servi à l'informateur de support d'interprétation de sa propre réalité sociale et de son parcours à travers la ville. (Alvir, 2013, p. 450)

Concernant la dimension esthétique, nos données ont révélé que les éléments du cadre bâti du quartier participent grandement à l'expérience quotidienne du quartier. Autant dans un quartier comme dans l'autre, les participant·e·s ont présenté des lieux qu'ils considéraient comme " beaux » (des éléments architecturaux, des espaces verts, des monuments symboliques) ou révélant une forme de soin à l'égard de leur quartier (des murales, des embellissements urbains). Ces espaces participent ainsi d'un sentiment de fierté des résidents et favorisent l'envie et la sensation de faire partie de la collectivité - à la fois celle du quartier et celle de la ville. Une participante exprime ainsi : " J'ai posté la photo et mes amis voient, écrivent les commentaires. Et là ils sont très intéressés. Je suis contente, et proud. » (Entretien VECSP, Photo 1).

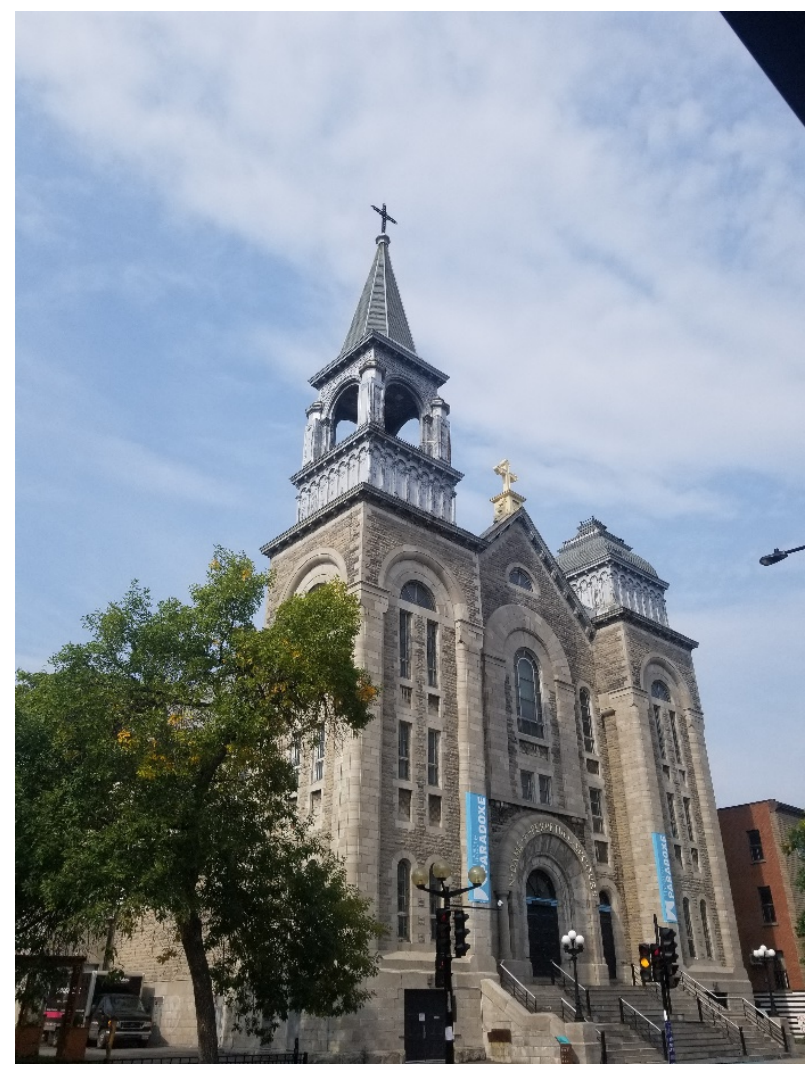

Photo 1

A contrario, les participant·e·s ont également photographié des espaces du quartier qu'ils jugent négligés (présence de déchets, manque d'entretien, une impression d'espaces publics délaissés). Ces photographies illustrent alors comment la sensation des participant·e.s du sentiment de marginalisation de leur milieu de vie s'étend à leur propre personne comme nouvelles arrivantes. Par exemple, un·e participant·e de MHM ne prend pas en photo son propre logement décrit comme " décrépi », mais appuie son propos en représentant un lieu qu'iel appelle la « maison hantée » et ajoute : « je me sens pas très bien avec moi-même de le dire, mais je n'ai pas le même niveau de vie que j'avais au Mexique. [...] c'est un peu : toi tu es un immigrant, alors n'importe quoi 
Goudet, Anna et Catherine Paquette. «Quand la « voix » des nouveaux·elles arrivant·e·s est exprimée et entendue grâce à la photographie : réflexions sur la méthode de recherche Photovoice ». Nouvelle Revue

va bien avec toi. " (Entretien MHM, Photo 2) Certains espaces jugés délaissés, inesthétiques ou « moins vivants " contribuent même au sentiment d'insécurité de certain·e·s participant·e·s. Les photographies et les entrevues ont donc permis de révéler la manière dont le soin apporté au quartier confère aux résidents le sentiment d'être pris en compte dans la collectivité au sens large. Or, la dimension esthétique est écartée, manquante dans le cadre des collectivités accueillantes.

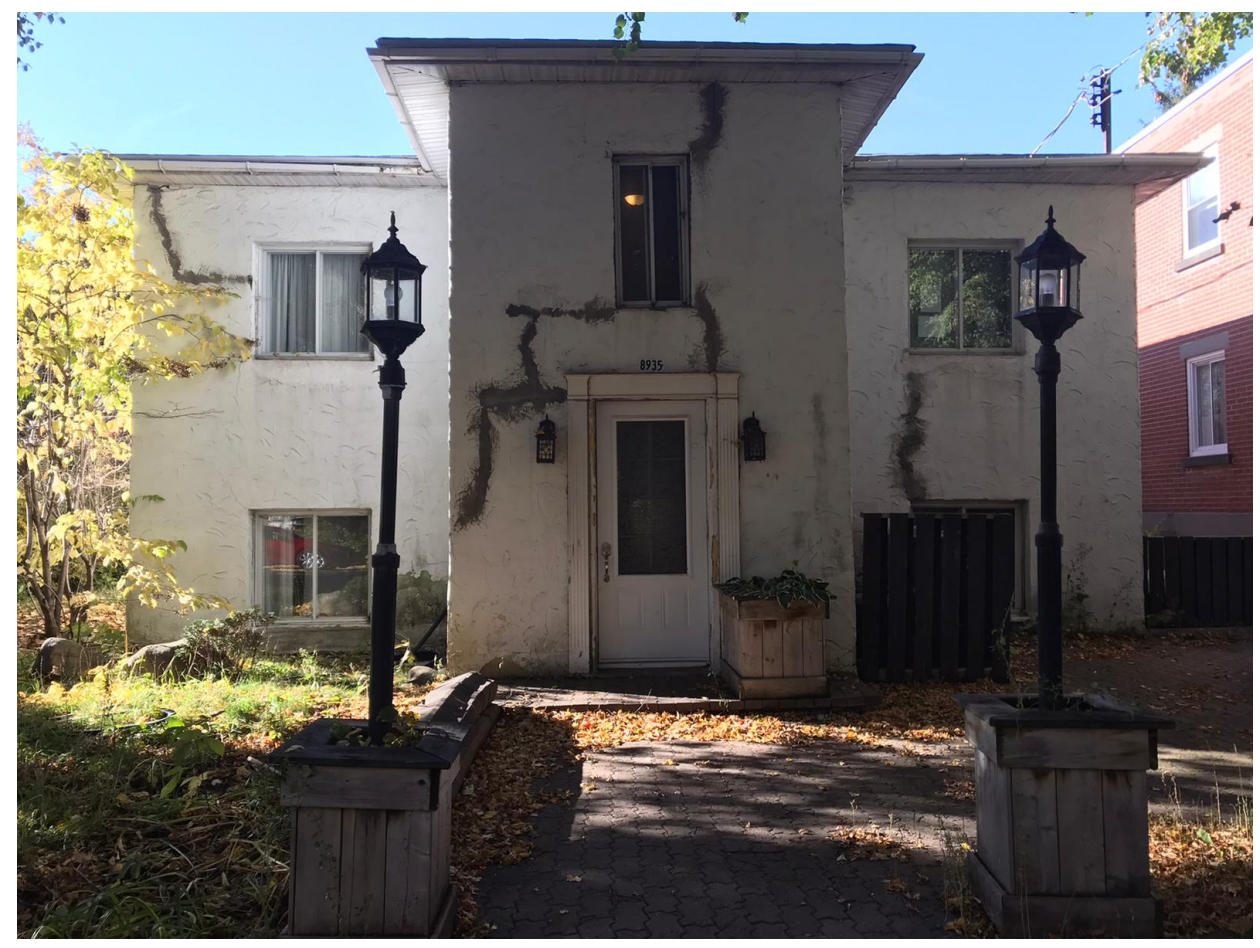

Photo 2

Concernant la dimension symbolique et émotionnelle de l'accueil, les participant·e·s ont fortement démontré qu'ils et elles avaient un attachement particulier à certains lieux de leur quartier. Ces espaces les ont marqué·e $\cdot s$ et sont devenus des repères dans leur vie quotidienne, en lien avec leur parcours migratoire et leur vie de famille. C'est par exemple le centre d'hébergement temporaire à l'arrivée en tant que demandeuse d'asile, le premier lieu connu en arrivant où on a pu parler sa langue natale - une boucherie, en l'occurrence -, l'école de quartier où ses enfants s'épanouissent, l'église où l'enfant a été baptisé, les rails de chemin de fer où on a de bons souvenirs lors de promenade avec la famille en visite. Sur ce dernier point, on constate que l'image fait émerger l'idée d'une sorte de " mémoire matérielle ») (Kwint et al., cité par Warren, 2005) incarnée pour eux·elles dans certains espaces de leur quotidien. Une participante raconte à propos de sa photo du port :« La première, c'est une photo du port. Pour moi c'est le lien entre le passé et ce que je suis maintenant. Parce que je travaillais beaucoup avec le commerce extérieur, mais je n'ai jamais travaillé près du port. [...] [rires] Mais maintenant c'est très présent dans ma vie actuelle. Et c'est pour ça que je l'ai pris, parce que je passe tous les jours ou presque face au port, et pour moi c'est comme un recordateur de qui je suis et d'où je viens [Chercheuse : Comment ça ?] c'est quelque chose qui me fait rappeler de qui je suis ou d'où je viens ou qu'est-ce que j'ai fait dans le passé, même si je le fais pas présentement » (Entretien MHM, Photo 3). 
Goudet, Anna et Catherine Paquette. « Quand la « voix » des nouveaux·elles arrivant·e·s est exprimée et entendue grâce à la photographie : réflexions sur la méthode de recherche Photovoice ». Nouvelle Revue

Synergies Canada, № 15 (2022)

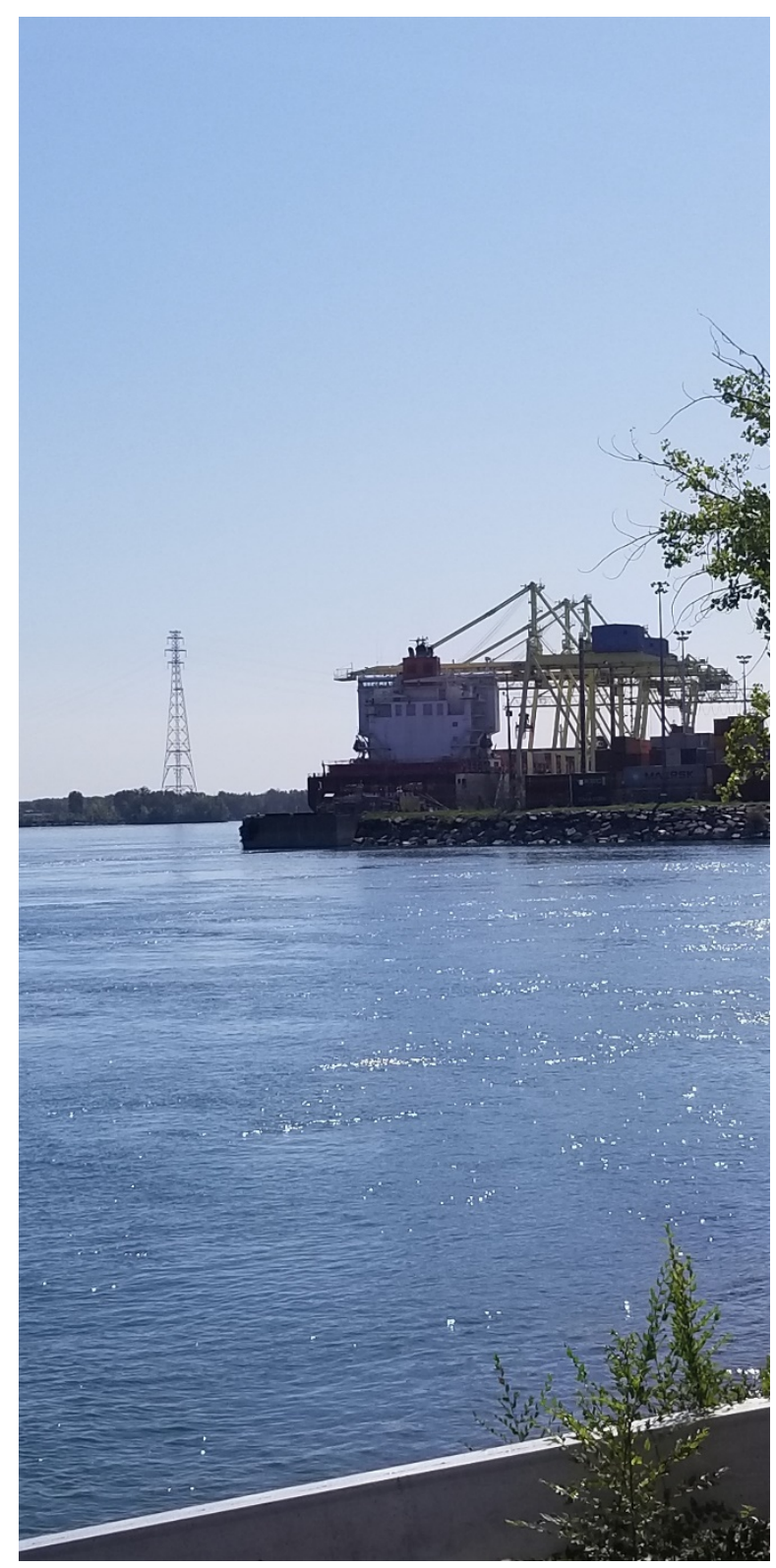

Photo 3

Le caractère symbolique de certains espaces révèle finalement la possibilité d'ancrage que le quartier peut offrir, en fournissant à ses résidents les moyens de se réaliser, de se reconnaître dans les espaces du quotidien, et de s'y projeter dans l'avenir. Comme dans la recherche d'Alvir (2013), photographier ces espaces a permis de donner aux participant·es un support pour discuter de leur expérience quotidienne dans leur quartier et des réflexions qu'évoquent certains lieux.

Ces nouvelles dimensions ayant émergé de la démarche des participant·e·s portent à croire que ces derniers ont pris une certaine place en tant qu'acteur-trice-s du processus de recherche, qu'ils et elles se sont approprié le thème de recherche. La prise de photographie, l'image, a permis de mettre à l'agenda, de « rendre visible » (Warren, 2005) certaines sensibilités au sujet d'espaces matériels, qui seraient fort probablement 
Goudet, Anna et Catherine Paquette. « Quand la « voix » des nouveaux·elles arrivant·e·s est exprimée et entendue grâce à la photographie : réflexions sur la méthode de recherche Photovoice ». Nouvelle Revue

restées dans l'ombre si notre démarche n'avait compris que des entretiens classiques. L'émergence de ces espaces dans la description de leur perception et expérience de l'accueil dans le quartier, grâce à la méthode Photovoice, permet d'approfondir notre questionnement sur l'accueil dans les quartiers et sur la notion de collectivité accueillante. La pertinence de certaines caractéristiques des collectivités accueillantes a été réaffirmée par des photographies et récits (non présentés ici), mais certaines limites de la notion apparaissent également. En effet, nous croyons à présent qu'elle se devrait d'inclure également les aspects esthétiques et symboliques du quartier qui relèvent de l'expérience individuelle et subjective des nouveaux arrivants. Ces résultats permettent également de consolider le fait que les collectivités accueillantes sont d'abord composées d'espaces qui répondent à un besoin d'appartenir des individus à leur environnement social, et d'en être reconnus. Rappelons donc à la lumière de ces résultats que l'expérience quotidienne vécue dans les lieux du quartier (qui comprend l'appréciation esthétique des lieux) contribue à la perception des participant·e.s de se trouver dans un quartier accueillant ou moins accueillant.

\subsection{Jouer avec l'image pour s'exprimer}

Des participant·e·s ont également joué avec l'ambiance autour des objets photographiés afin d'accentuer la portée de leur commentaire au sujet d'aspects positifs ou négatifs de leur quartier. Par exemple, la photographie 8 montre un parc qu'une participante trouve mal entretenu. Prise lors d'une journée au temps maussade, la participante souligne comment l'image permet de donner un côté « dramatique » au parc : "C'est très populaire ce parc, il y a beaucoup de familles qui habitent ici, c'est le plus proche, mais je le trouve sale, je le trouve toujours... je trouve pas le mot, mais même le sable qui doit être dedans est partout. Je pense que c'est complètement oublié. Et le ciel hier était tout gris, alors c'est trop dramatique pour moi, je pense qu'il a besoin un peu d'amour. " (Entrevue MHM, photo 4). Ce terme " dramatique » fait ici référence à l'agencement de l'ambiance projetée par l'image et de la déception de la participante quant à l'entretien du parc - une description dont la sensibilité n'aurait sans doute pas autant émergé lors d'un entretien sans cette représentation visuelle, qui prend ici le rôle de métaphore, et qui permet à la participante de s'exprimer alors qu'elle « ne trouve pas le mot $»$.

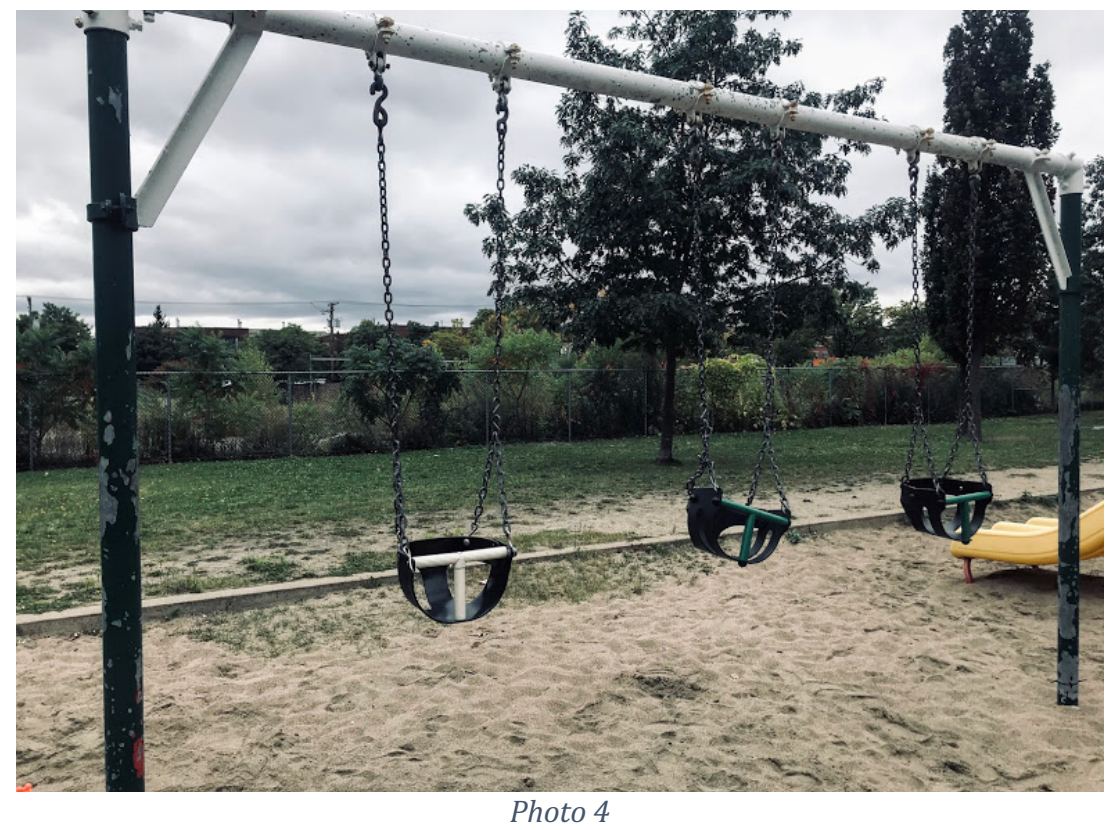

D'autres participant $\cdot e \cdot s$ orientent leur angle de vue durant la prise de photo pour refléter et accentuer leur propos. C'est le cas notamment d'une photo d'école, considérée comme un repère dans le quartier. La photo est prise depuis la cour de récréation (photo 5) qui est refaite à neuf récemment, comme sa fille qui y est une nouvelle élève : « Justement ce morceau de l'école [pris en photo] était pas construit, c'est tout neuf, c'est pour ça que j'ai pris la photo à cet endroit parce que c'est tout neuf, comme elle à l'école cette année ! » (Entrevue MHM, photo 5) 
Goudet, Anna et Catherine Paquette. « Quand la « voix » des nouveaux·elles arrivant·e·s est exprimée et entendue grâce à la photographie : réflexions sur la méthode de recherche Photovoice ». Nouvelle Revue

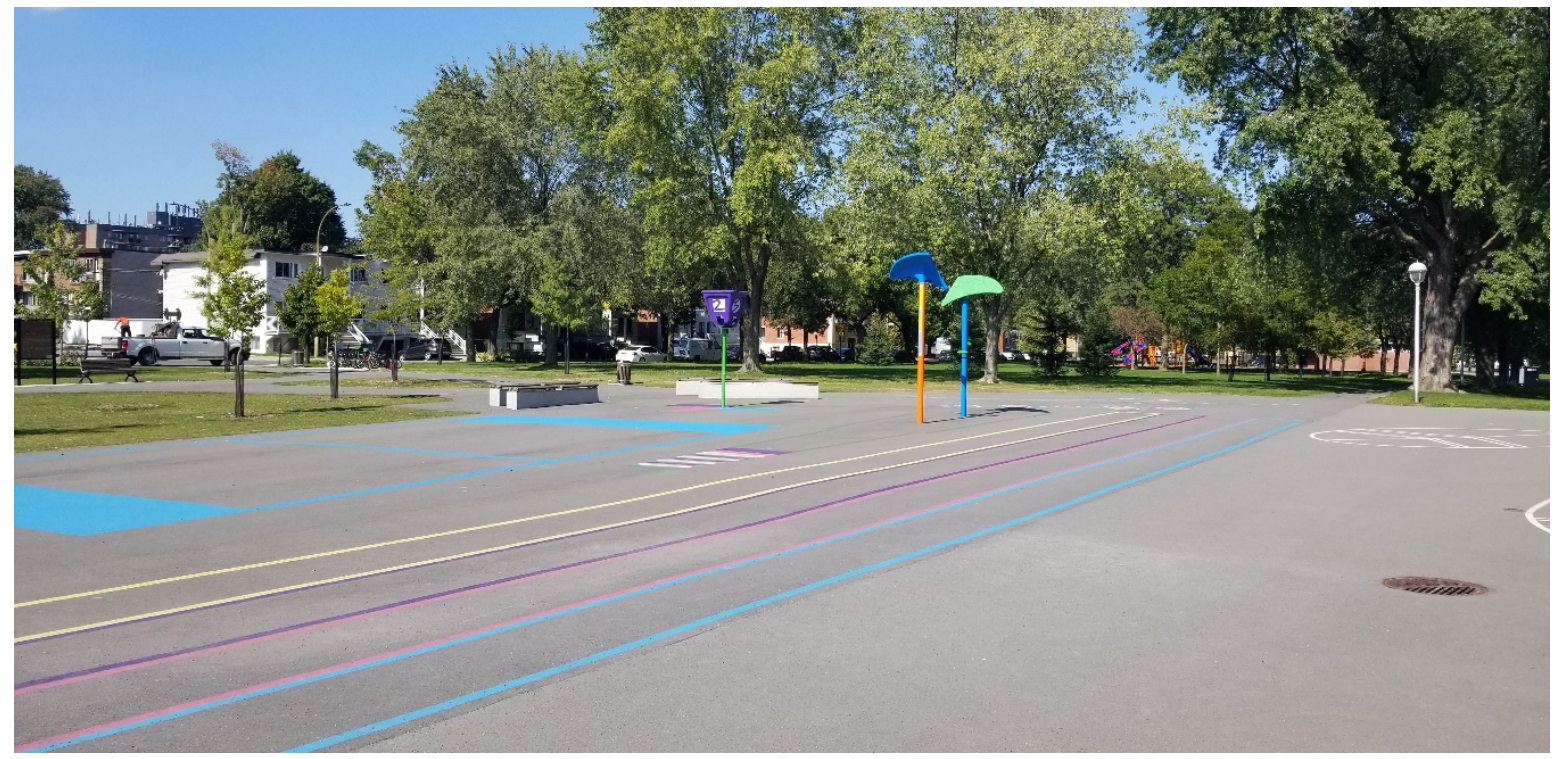

Photo 5

\section{Co-construction et apports pour les participants eux-mêmes}

Les participant $\cdot e \cdot s$ ont eu différentes réactions vis-à-vis de leur propre démarche de prise de photos, ils et elles se sont approprié la démarche de différentes manières. Par ailleurs, à partir d'une analyse des discours tenus en entretien, nous constatons qu'en s'appropriant la méthode de recherche, certaines personnes se sont aussi approprié différemment le terrain de la recherche : leur quartier, leur milieu de vie. Elles ont « vu autrement » leur environnement quotidien, et par là s'est créé « un nouveau rapport de proximité/distance » avec ce qui les entourait, comme le remarque Leroy (2020) dans son recours à la méthode photographique pour saisir les représentations du plurilinguisme de jeunes dans le Sud italien.

En ce qui concerne l'attitude par rapport à la prise de photographie, l'une des participantes a employé le terme de «reportage » pour désigner son ensemble de photos, montrant fièrement chaque aspect de sa vie quotidienne, ce qui laisse croire à une certaine agentivité perçue par cette personne dans la démarche ; elle était devenue reporter d'un jour, parcourant le quartier afin d'en montrer une représentation fidèle à son expérience personnelle et pouvant être rendue publique. La participante, qui est une entrepreneure, a d'ailleurs souligné au moyen de plusieurs photographies l'importance d'amener certains changements dans le quartier, auxquelles elle compte contribuer en lançant prochainement son entreprise - tant le corpus de photos que sa façon de le décrire reflètent cet aspect de sa personnalité. Un autre participant, employant une approche différente, a plutôt souhaité présenter de façon "objective " les aspects positifs et négatifs de son quartier, en constituant une sorte de " guide à l'intention de futurs nouveaux-elles arrivant·e.s ». Ces deux postures, très différentes, montrent que la démarche peut être comprise de façon différente par chaque participant·e.s et leur laisser la liberté de s'exprimer au sujet de leur quartier via la photo, mais aussi au sujet de leur prise de photos.

En parallèle à l'expression de leur voix et opinion, il semble que les participant·e.s aient approfondi leurs connaissances du quartier et leur appropriation de celui-ci. Jumelant la documentation de leurs espaces quotidiens et la réflexion sur leur expérience dans ces espaces, la prise de photographie a fait émerger chez les participant·e·s des commentaires montrant qu'ils avaient eux·elles-mêmes découvert ou redécouvert certains éléments du quartier. C'est ainsi qu'une participante a révélé, lors de l'entretien, avoir fait des recherches en ligne au sujet de ses espaces photographiés, découvrant entre autres que l'église, qu'elle appréciait pour son architecture, est en fait une salle de concert qu'elle pourrait fréquenter lors d'évènements culturels (photo 1).

L'exercice photographique a aussi permis à un participant de mieux cerner « sa » frontière du quartier (dont on 
Goudet, Anna et Catherine Paquette. « Quand la « voix » des nouveaux·elles arrivant·e·s est exprimée et entendue grâce à la photographie : réflexions sur la méthode de recherche Photovoice ». Nouvelle Revue

avait discuté dans l'entrevue préliminaire) : « J'avais repensé à ce qu'on avait pu dire la dernière fois et c'est pour ça que j'ai pris [cette photo]. En fait, ça, ça serait une bonne illustration de ma frontière, parce qu'en fait là on est sur Viau et Lafontaine, et en fait moi je me limite toujours à cette partie-là, et puis après là on est sur toute l'extrémité où j'avais jamais mis les pieds. J'ai toujours pas mis les pieds là-bas, mais au moins j'ai identifié que là ça fait effectivement une frontière. " (Entrevue MHM). Pour un autre, cela a été l'occasion justement de repousser les frontières de son milieu de vie en allant explorer une partie jusqu'alors moins connue du quartier : « Je me suis dit ça sera l'occasion d'aller voir un peu le quartier à côté, je connais un petit peu mais moins le côté Sainte Catherine/Ontario. [...] c'était un peu pour les photos aussi [rires] » (Entrevue MHM).

L'exercice a également été pour certain·e·s une occasion d'introspection. La possibilité, ou la contrainte, d'utiliser une caméra pour explorer son quartier amène à se questionner aussi soi-même. Pour un participant, le parcours de prise de photographie a permis de réfléchir à « l'histoire qu'il se raconte » (Entrevue MHM) au sujet du cadre bâti et de la réputation du quartier, en lien avec sa propre position d'immigrant et son parcours de vie au sein de la communauté locale. Notons que cette réflexion a aussi été possible, ou encouragée, par le fait que notre démarche comprenait deux rencontres entre lesquelles les participant·e·s avaient le loisir de réfléchir aux propos qu'ils·elles allaient transmettre lors de l'entretien à venir.

Nykiforuk et ses collègues ont noté le même phénomène lors de leur étude dans un quartier albertain : « In the current project, participants noted that they learned about their community while participating in the project. " (Nykiforuk et al. , 2011, p. 16). Le processus de la prise de photographie contribue lui-même à une introspection qui peut mener à un changement dans la participation ou dans la volonté de contribuer au changement social au niveau local: "Through the process of taking photos, participants intuitively - and explicitly - reconsider issues that may lead to change in self and foster an impetus to participate in activities that stimulate action or create social change at the community level. » (Nykiforuk et al. , 2011, p. 119).

Nos résultats portent à croire que les participant·e·s ont été pleinement acteur·trice·s de la représentation qu'ils-elles voulaient faire de leur quartier et de leur vie quotidienne lors de ce processus - et que bien entendu ce discours a tout de même pu être influencé ou orienté par certaines consignes ou questions lors des rencontres avec les chercheures. La démarche peut avoir pour effet non seulement de se sentir engagé·e dans le processus de recherche, mais également d'approfondir le sentiment d'appartenance envers le quartier à travers l'action de le photographier et de le raconter, d'en apprendre plus sur ses espaces, ainsi que faire émerger un processus réflexif sur son parcours migratoire.

\section{Défis de la méthode}

Les entretiens ont aussi révélé des indices d'une relation d'enquête particulière et de différents rapports à la photographie également. Rappelons que la situation de l'entretien est incontestablement teintée par la relation entre les chercheuses (étudiantes, femmes, blanches, francophones) et les participant·e.s, dont les caractéristiques hétérogènes peuvent aussi nourrir différents rapports. Mais plus encore, des défis sont aussi apparus dans cette relation en lien avec la méthode Photovoice. Entre autres, nous avons noté chez plusieurs participant·e.s une volonté de présenter des photographies qui plaisent, visuellement, aux chercheuses. Plusieurs personnes ont exprimé ce désir de plaire en suggérant aux chercheuses de choisir les photographies qu'elles trouvaient jolies, et de laisser de côté les autres.

Pour d'autres, nous avons remarqué que l'aspect esthétique s'est transformé en frein pour présenter des espaces qu'ils jugent pourtant fortement significatifs dans leur vie quotidienne ou parcours d'établissement : « $Y$ en a des endroits qui m'ont marqué, comme le PITREM [un organisme communautaire du quartier] par exemple, mais ça me gênerait de prendre des photographies de ça, disons que ça serait moins artistique alors ça me dérangerait un peu de le faire » (Entrevue MHM). Par ailleurs, on peut redouter une forme de reproduction de la disqualification sociale dans l'interaction de la recherche : le fait de posséder ou non un appareil photo, et la qualité des photos liées au type d'appareil, peuvent être un obstacle, d'abord, à la participation, mais aussi se transformer en gêne pendant la participation. Certains ont aussi déclaré s'être « mis des limites » dans l'exercice photographique : " j'aime vraiment les photos de rue résidentielle. Parce que je trouve que ça donne un peu un aperçu du calme. $Y$ a aussi le fait que j'ai du mal à prendre des photos dans une rue où y a beaucoup de passages, parce que je me vois mal m'immobiliser devant un truc et prendre une photo comme ça, enfin je me mets des limites tout seul, mais c'est ça quoi. » (Entrevue MHM).

Enfin, le médium photographique, en favorisant grandement l'expression de dimensions relevant du mode de 
Goudet, Anna et Catherine Paquette. « Quand la « voix » des nouveaux·elles arrivant·e·s est exprimée et entendue grâce à la photographie : réflexions sur la méthode de recherche Photovoice ». Nouvelle Revue

vie et de la sensibilité des individus a certainement aussi limité la possibilité de rendre visible d'autres dimensions plus systémiques, telles que les opportunités d'emploi ou d'éducation, la justice sociale, la discrimination, etc. Cela est accentué également par l'échelle du quartier. Les entretiens ont aussi laissé croire que les participant $\cdot e \cdot s$ avaient tendance à présenter davantage en photo les aspects positifs que négatifs de leur milieu de vie. C'est par les questions posées en entrevue que les dimensions plus néfastes sont apparues, par exemple les difficultés de créer des liens avec des personnes résidant dans le quartier, ou encore des commentaires racistes entendus dans certains lieux associatifs.

Par conséquent, il serait naïf de croire que la prise de photo peut à elle seule permettre d'aborder tous les aspects de la vie des participant·e·s. Dans le cadre du projet de recherche-action réalisé en collaboration avec les organismes communautaires, ce genre de remarque nous a amenés à nuancer les résultats et à souligner que le choix de certain'e-s participant·e.s d'occulter certains lieux ou dynamiques du quartier ne doit pas être compris comme l'absence de fréquentation de ces lieux, ni comme l'absence de dynamiques d'exclusion, par exemple.

À la suite des entretiens et des analyses, reste encore le défi de faire voyager la voix des participant·e.s dans les différentes sphères de la vie publique. Il est à la fois important de saisir et de conserver le sens attribué par la personne au sujet de l'image, en plus de savoir créer les espaces nécessaires à la bonne réception de cette parole dans une perspective de changement social. Lors de la diffusion, il ne s'agit pas seulement de souligner que différentes personnes peuvent avoir différentes perceptions d'une même image, mais bien de mettre en exergue l'esprit et le contexte dans lequel la photo a été prise par le.la participant $\cdot e$ afin que sa voix soit entendue (Warren, 2005, p. 873). Warren souligne enfin que la présence des photos des participant·e·s dans les communications subséquentes au projet est importante afin de convier et représenter leur voix telle que ces participant $\cdot e \cdot s$ ont souhaité l'exprimer visuellement.

\section{Conclusion}

Cet article a permis d'aborder certains avantages et défis apportés par la méthode Photovoice pour l'expression de personnes immigrantes dans la recherche sociale en milieu urbain. Dans le cadre de notre projet, les participant·e·s ont exprimé des enjeux différents de ceux habituellement évoqués dans la littérature sur les collectivités accueillantes, tels que les aspects esthétiques et symboliques de certains espaces. Grâce au médium photographique, nous avons en effet pu cerner comment le sentiment de fierté vis-à-vis les espaces jugés esthétiquement beaux dans son quartier participe aussi de l'expérience et du bien-être de personnes immigrantes dans les premières années de leur installation (en plus de ressources nécessaires à l'accueil et l'établissement des nouveaux $\cdot$ elles arrivant·e-s). Ensuite, il a été noté que plusieurs personnes rencontrées ont adapté la méthode Photovoice à leur guise, en fonction de leurs différentes intentions et sensibilités. Durant la démarche, certaines personnes ont même poursuivi leur réflexion au sujet de leur quartier ou accumulé de nouvelles connaissances à son sujet, ce qui laisse croire que la démarche a participé à leur appropriation de leur milieu de vie.

Au-delà de ces constats portant sur la relation d'enquête dans la méthode Photovoice, nous soumettons la nouvelle hypothèse que la méthode Photovoice permettrait de faire émerger différemment la voix des personnes immigrantes, autrement plutôt entendues dans l'espace public autour de questions sur leur «insertion» et leur usage des ressources d'aide. La méthode Photovoice pourrait faire l'objet de plus amples réflexions sur la capacité des personnes migrantes à exprimer non seulement leurs besoins, mais également leur voix en tant qu'individus aux personnalités et sensibilités uniques, dans un contexte où certains discours publics à leur endroit sont situés dans des registres utilitaristes, voire ouvertement hostiles. Mais déjà le projet a porté ses fruits dans les quartiers ciblés : après une présentation des résultats auprès des membres de l'un des comités, ceux-ci se sont emparés du projet pour organiser une exposition des photographies afin de sensibiliser les

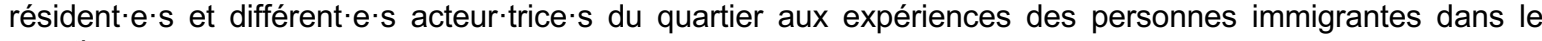
quartier.

\section{Notes}

${ }^{1}$ Dans cet article nous choisissons d'utiliser le terme « immigrant », car nous reprenons le terme courant utilisé par les organismes avec qui nous avons collaboré - en plus d'être le terme dans lequel se sont reconnues les personnes qui ont accepté de participer à la recherche. 
Goudet, Anna et Catherine Paquette. « Quand la « voix » des nouveaux·elles arrivant·e·s est exprimée et entendue grâce à la photographie : réflexions sur la méthode de recherche Photovoice ». Nouvelle Revue Synergies Canada, № 15 (2022)

\section{Bibliographie}

Alvir, S. (2013). Quelles dynamiques spatiales et sociolangagières dans l'appropriation des espaces urbains par les résidents étrangers à Lausanne?: quand la photographie donne à voir les indices de cette appropriation. [Thèse de Doctorat, Université de Fribourg]. Récupéré de: http://doc.rero.ch/record/210346

Budach, G. et Patrick, D. (2011). Donner une voix aux Inuit urbains : "Photovoice » comme une pratique de multilittératie dans la construction d'identité et de savoirs transfrontaliers. Cahiers de I'ILOB, 2. https ://doi.org/10.18192/olbiwp.v2i0.1083

Belkhodja, C. (2009). Toward a more welcoming community? Observations on the Greater Moncton Area. [en ligne] Disponible sur: http://citeseerx.ist.psu.edu/viewdoc/download;jsessionid=7E7AB650B1D0FB70C7D1C72D11710933 ?doi=10.1.1.545.9884\&rep=rep1\&type=pdf

Désilets, G. et Goudet, A. (2019). La mise en œuvre des politiques d'accueil des migrants à l'échelle des quartiers montréalais : l'étude de l'initiative Vivons nos quartiers. Lien Social et Politique, 83, 230-248.

Esses, V. M., Hamilton, L. K., Bennett-Abuayyash, C. et Burstein, M. (2010). Characteristics of a welcoming community. Citoyenneté et Immigration Canada. http://p2pcanada.ca/wpcontent/uploads/2011/09/Characteristics-of-a-Welcoming-Community-11.pdf

Evans-Agnew, R. A. et Rosemberg, M.-A. (2016). Questioning Photovoice research : Whose voice?. Qualitative Health Research, 26(8), , 1019-1030. doi : 10.1177/1049732315624223

Goudet, A., Paquette, C. et charrette, A. (2021). Phase 2 : Documenter, contextualiser et valoriser l'initiative "Vivons nos quartiers ». Retour sur l'histoire de l'accueil, la concertation locale et les représentations des espaces accueillants pour les nouveaux arrivants. Rapport BMRC-IRMU. https://bmrcirmu.info.yorku.ca/files/2021/02/Vivons-Nos-Quartiers-II Final 9Feb2021.pdf?x15611

Houle, J., Coulombe, S., Radziszewski, S., Boileau, G., Morin, P. Leloup, X., Bohémier, H. et Robert, S. (2016). Public housing tenants' perspective on residential environment and positive well-being: An empowerment-based Photovoice study and its implications for social work. Journal of Social Work, 18(6), 703-731,. doi:10.1177/1468017316679906

Leroy, M. (2020). "C'est mon quartier. C'est tout. Là tout est multilingue » Approcher les imaginaires du plurilinguisme de jeunes sud-tyroliens par la photo. Éducation et sociétés plurilingues, 48, 51-64.

Lofton, S. et Gran, A. K. (2020). Outcomes and intentionality of action planning in Photovoice: A literature review. Health Promotion Practice, 22(3), 318-337. doi : 10.1177/1524839920957427

Nykiforuk, C. I. J., Vallianatos, H. et Nieuwendyk, L. M. (2011). Photovoice as a method for revealing community perceptions of the built and social environment. International Journal of Qualitative Methods, 10(2), 103-124, doi:10.1177/160940691101000201

Payet, J.-P., Giuliani, F. et Laforgue, D. (2008). La voix des acteurs faibles. De l'indignité à la reconnaissance. Presses universitaires de Rennes.

Ronzi, S., Pope, D., Orton, L. et Bruce, N. (2016). Using Photovoice methods to explore older people's perceptions of respect and social inclusion in cities : Opportunities, challenges and solutions. SSM Population Health, vol. 2, 732-745. https://doi.org/10.1016/j.ssmph.2016.09.004

Valiquette-Tessier, S.-C., Vandette, M.-P. et Gosselin, J. (2015). In her own eyes : Photovoice as an innovative methodology to reach disadvantaged single mothers. Canadian Journal of Community Mental Health, 34(1), 1-16. https ://doi.org/10.7870/cjcmh-2014-022 
Goudet, Anna et Catherine Paquette. « Quand la « voix » des nouveaux·elles arrivant·e·s est exprimée et entendue grâce à la photographie : réflexions sur la méthode de recherche Photovoice ». Nouvelle Revue

Synergies Canada, № 15 (2022)

Wang, C. et Burris, M. A. (1997). Photovoice : Concept, methodology, and use for participatory needs assessment. Health Education \& Behavior, 24(3), 369-387. https ://doi.org/10.1177/109019819702400309

Warren, S. (2005). Photography and voice in critical qualitative management research. Accounting, Auditing \& Accountability Journal, 18(6), 861-882. https://doi.org/10.1108/09513570510627748 